\title{
The impact of clinically undiagnosed injuries on survival estimates
}

\author{
Rolf Gedeborg, MD, DrMedSc; Ingemar Thiblin, MD, PhD; Liisa Byberg, MSc, PhD; Lisa Wernroth, MSc; \\ Karl Michaëlsson, MD, PhD
}

\begin{abstract}
Objectives: Missed injury diagnoses may cause potentially preventable deaths. To estimate the effect of clinically undiagnosed injuries on injury-specific survival estimates and the accuracy of an injury severity score. To also estimate the potentially preventable mortality attributable to these injuries.

Design, Setting, and Patients: In a nation-wide, populationbased study, data were collected from all hospital admissions for injuries in Sweden between 1998 and 2004. We studied 8627 deaths in hospital among 598,137 incident hospital admissions.

Measurements and Main Results: New specific-injury categories were added in 7.4\% (95\% confidence interval [CI] 6.8-8.0) of all deaths with an autopsy rate of $24.2 \%$. It was estimated that this proportion would have increased to $25.1 \%$ (95\% CI $23.0-$ 27.2), if all deaths had been autopsied. The most pronounced effect of clinically undiagnosed injuries was found for internal organ injury in the abdomen or pelvis, where they reduced the
\end{abstract}

estimated survival from 0.83 to $0.69(95 \%$ CI for the difference: 0.09-0.20). Autopsy diagnoses also revealed substantial bias of survival estimates for vascular injuries in the thorax and crush injuries to the head. The performance of the International Classification of Diseases Injury Severity Score improved when autopsy diagnoses were added to hospital discharge diagnoses. The maximum proportion of injury deaths attributable to missed injuries was estimated to be $6.5 \%$.

Conclusions: Maintaining a high autopsy rate and merging accurate hospital discharge data and autopsy data are effective ways to improve the accuracy of survival estimates and mortality prediction models, and to estimate mortality attributable to diagnostic failures. (Crit Care Med 2009; 37:449-455)

KeY WoRDS: traumatology; outcome assessment; autopsy; survival; registries

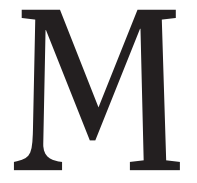

onitoring the burden of injury to patients and to society is an important public health issue. Although studies based on trauma center populations and specialized trauma registers can provide important information regarding trauma care, the external validity of the results may be questioned on grounds of regional differences and selection bias $(1,2)$. Population-based studies may be more relevant from a public health perspective. Regardless of the study type there is need for reliable categorization of injuries and for accurate estimates of injury severity to evaluate outcome.

From the Department of Surgical Sciences, Units for Anesthesiology and Intensive Care (RG); Forensic Medicine (IT); Orthopedics (LB, KM); Uppsala University Hospital, Uppsala, Sweden; and Uppsala Clinical Research Center (RG, LW, KM), Uppsala University Hospital, Uppsala, Sweden.

Supported, in part, by The Laerdal Foundation for Acute Medicine, The Swedish Society of Medicine, and The Swedish Research Council.

The authors have not disclosed any potential conflicts of interest.

For information regarding this article, E-mail: rolf.gedeborg@surgsci.uu.se

Copyright ( 2009 by the Society of Critical Care Medicine and Lippincott Williams \& Wilkins

DOI: 10.1097/CCM.0b013e318194b164
Survival risk estimates for different injury categories and injury severity scores such as the International Classification of Diseases Injury Severity Score (ICISS) rely on accurate classification of patients based on their injury diagnoses (3-5). It is a well-known problem that injuries can be overlooked clinically and remain undetected unless autopsy is performed (68). Clinically undiagnosed injuries may not only indicate deficiencies in the trauma care system, but can also be a consequence of inaccuracy in discharge coding of injuries. Irrespective of the reason, failure to identify all significant injury diagnoses can potentially lead to substantial misclassification of deaths, resulting in underestimation of injuryspecific mortality risks and consequently leading to bias in mortality prediction models.

By merging results from autopsy with hospital discharge diagnoses, the accuracy of survival risk estimates could be increased. The Swedish unique personal identification numbers enable reliable matching of hospital discharge records with autopsy data for the entire Swedish population. This allows quantification of the impact on survival estimates when autopsy diagnoses are added to hospital discharge diagnoses. It is also of interest to try to estimate the potentially preventable mortality associated with missed diagnoses.

The principal aim of this study was to assess the impact of adding autopsy diagnoses to hospital discharge diagnoses on injury-specific survival risk estimates and on the predictive capacity of ICISS scores. A secondary objective was to evaluate the ability of different patient characteristics to predict both whether autopsy would be performed and whether new injuries would be found. This would allow assessment of the relation between autopsy rate and the number of new injuries discovered at autopsy. Together with predicted survival from ICISS, this would also allow estimation of the excess mortality associated with clinically undiagnosed injuries.

\section{MATERIALS AND METHODS}

Study Population and Setting. All hospital admissions for injury during the years 19982004 were extracted from the Swedish Hospital Discharge Register. This is a complete national register maintained by the Swedish National Board of Health and Welfare and covers all inpatient care in Sweden. The sole purpose of collecting these data is future research and there is no connection with insurance claims or reimbursement. The register includes information about the main diagnosis, 
comorbidity (up to seven secondary diagnoses), and cause of injury. Since 1997 the diagnoses have been coded according to the International Statistical Classification of Diseases and Health Related Problems, 10th revision (ICD-10).

We defined a hospital admission for injury as having a main diagnosis from chapter XIX in ICD-10, but we excluded allergy (T78) and complications of surgical or medical care (T80-T88 or Y40-Y84). Late effects of injury (T90-T98 or Y85-Y89) were also excluded. All records that represented direct transfers from another department or hospital were linked to the original injury admission record using the personal identification number and admission/discharge date. Injury admissions were classified as readmissions a) if they were planned; b) if the patient had been admitted primarily to a rehabilitation facility; and/or c) if the patient had had a previous injury admission within 360 days with the same main diagnosis. Readmissions were excluded, leaving only incident injury admissions for analysis. These patients were admitted to 112 different hospital facilities located in all of the 21 counties (administrative healthcare regions) of Sweden. The study was approved by the regional Human Ethics Committee.

Cause of Death Data. The National Cause of Death Register is maintained by the Swedish National Board of Health and Welfare and contains information on all deaths in Sweden. If autopsy has been performed, it is classified as either clinical or forensic. Records from the cause of death register were matched to records from the hospital discharge register through the personal identification number.

Classification of Injury Diagnoses. ICD-10 is a valuable tool for coding of injuries, but there are too many ICD-10 diagnoses for them to be used for descriptions of mixed injury populations. To reduce the numerous ICD-10 diagnoses to a more reasonable number of diagnostic categories, the ICD-10 injury mortality diagnosis matrix was developed by the National Center for Health Statistics, Centers for Disease Control, USA (5). This matrix categorizes individual injury diagnosis codes by body region and nature of injury. The matrix was modified in this study to include the additional diagnoses $\mathrm{T} 00[.0-.1, .6]$, T01[.0-.1, $.6, .8]$, Т02[.0, .6-.7], Т03[.0, .4], ], T04[.0, .4, $.7]$, T05[.1, .4, .6], T06[.0-.1, .8], and T29[.0.7], to make it fully compatible with the Swedish clinical modification of ICD-10. The injury categorization was done twice for each admission; first using only hospital discharge diagnoses and then using both these diagnoses and additional diagnoses identified at autopsy. Injuries classified in the injury matrix as "multiple injuries" and "unspecified injury" and/or as "multiple body regions" and "unspecified" were considered to be unspecific diagnoses, and all others to be specific. Causes of injury were classified according to the matrix developed by the National Center for Health Statistics, Centers for Disease Control, USA $(9,10)$.
Statistics. Survival risk was calculated for each injury category in the ICD-10 injury mortality diagnosis matrix using a) hospital discharge diagnoses alone and b) both hospital discharge diagnoses and autopsy diagnoses. The risk difference between these two procedures was then calculated for each category. A 95\% confidence interval (CI) was calculated for each risk difference, using a bootstrap procedure with 1000 replications allowing for the dependence among the survival risk estimates.

Calculation of Injury Severity. The ICISS has been shown to perform well compared with other injury severity scores $(3,4)$. It is calculated on the basis of survival risk ratios (SRRs) for individual injury ICD-10 codes (4). This ratio represents the proportion of patients with a specific-injury code who survived to hospital discharge.

To assess the impact of autopsy diagnoses on the predictive capacity of ICISS for hospital mortality, two different sets of SRRs were calculated using data from the period 1998 through 2002. One set was based only on hospital discharge diagnoses, and the other was derived from both hospital discharge diagnoses and autopsy diagnoses. For assessment of their predictive capacity, these SRRs were used to calculate ICISS on injury events during the period 2003-2004. The ICISS score (survival probability) for the individual patient was calculated as the product of each of the SRRs corresponding to the patient's injuries (i.e., the product of the probabilities of surviving each of their injuries individually). The ability of ICISS to predict hospital mortality was assessed in logistic regression models adjusted for age and sex. Age and ICISS were entered as restricted cubic splines in these models. The area under the receiver operating characteristic curve (c-statistic) was calculated as a measure of predictive capacity. A 95\% CI for the difference in c-statistic was calculated using bootstrap samples from the original dataset with 1000 replications and the percentile method.

ICISS was also used to adjust for injury severity in regression models. For this application SRRs were derived from the entire study population, by only using hospital discharge diagnoses. A variable was also created for the number of severe trauma cases at each hospital. Severe trauma was defined as ICISS $<10$ th percentile $(0.9367)$.

Prediction Models. Two different prediction models were developed. One was intended for prediction of whether autopsy would be performed, and the other for prediction of the probability that a new specific injury would be diagnosed at autopsy. The same variables were used in the models and were selected on the basis of clinical reasoning. Age, sex, injury categories, ICISS (as a restricted cubic spline), length of hospital stay (after log transformation), cause of injury, intent of injury, and geographical region were used in logistic regression modeling. Binary indicator variables for injury categories that generated unstable parameter estimates (very large standard errors) were removed from the models. This instability was due to empty cells in the contingency table, rareness of injuries, or collinearity (when there was an injury category identical to a cause of injury category, e.g., poisoning and burn). Collinearity was assessed from variance inflation factors.

We applied the above two prediction models to estimate the number of new diagnoses that would be found if more cases of hospital death underwent autopsy. All deaths not subjected to autopsy were, therefore, ranked in descending order according to their estimated probability of being subjected to autopsy. The mean of the predicted probabilities of finding a new specific diagnosis at autopsy was calculated for each centile and plotted against the autopsy rate. A 95\% CI for the estimated proportion of deaths in which a new specificinjury category would be found if all deaths were autopsied was derived using a bootstrap procedure with 1000 replications and the percentile method.

Attributable Proportion of Mortality. The excess mortality attributable to clinically undiagnosed injuries was estimated from the predicted survival for the deaths where missed injuries were identified at autopsy. Their predicted survival (ICISS) was calculated on the basis of all their injuries, including autopsy diagnoses. Two different sets of SRRs were again used. One was based on hospital discharge data only, and the other was derived from combining hospital discharge data with autopsy diagnoses. The first set of SRRs reflects the prognosis if the patients had had their injuries diagnosed clinically. The second set of SRRs reflects the prognosis if they had had at least one clinically undiagnosed injury, assuming that no patients with such missed injuries survive. This latter assumption is necessary, because it is not possible to estimate the number of patients surviving with a missed injury diagnosis. The estimated impact of missed injuries on predicted survival is, therefore, a worst case scenario. The increase in predicted mortality risk ( 1 - median ICISS) associated with missed injuries is used to estimate a relative risk. This is calculated using all injuries (including autopsy diagnoses) as mortality risk predicted from SRRs based on both hospital and autopsy data divided by mortality risk predicted from SRRs based on hospital data only. By use of this quotient, the proportion of mortality attributable to missed injuries can be calculated as $(1$ - relative risk)/relative risk. A $95 \%$ CI for the attributable proportion was calculated using bootstrap samples from the original dataset with 1000 replications and the percentile method.

The SAS version 9 (SAS Institute, Cary, $\mathrm{NC)}$ statistical package and the R statistical package (11) were used for data management and statistical analyses. 


\section{RESULTS}

During the 7-year study period there were 598,137 incident injury admissions with a main diagnosis of injury to 112 different hospitals, and 8627 of these patients died in hospital (1.4\%). Of these hospital deaths, $24.2 \%$ underwent autopsy. This autopsy rate was notably influenced by the 3798 deaths (44.0\%) associated with hip fractures. When hip fractures were excluded, the autopsy rate increased to $35.2 \%$. Baseline characteristics by autopsy status are shown in Table 1. In a multivariable logistic regression model, every 10 years' increase in age was associated with an approximate 38\% reduction of the probability that autopsy would be performed (Table 2). When the cause of injury was cut/pierce, fire/flame, firearm, struck by/against blunt objects, or traffic, this was independently associated with an increased probability of un- dergoing autopsy. The intent of injury was also predictive, in the sense that homicide or an undetermined intent was associated with a higher probability of autopsy. The discriminative capacity of this multivariable model was high. The c-statistic (area under the receiver operating characteristic curve) was 0.854 , which means that for $85 \%$ of the cases, the probability that autopsy would be performed, as predicted by the model, was higher in a patient subjected to autopsy than in a nonautopsied patient.

Among the 2084 patients who died in hospital and underwent autopsy, new injury categories were found in 807 patients. Of these, $79.1 \%$ (638 of 807 ) were related to specific-injury categories, whereas the rest were unspecific and therefore uninformative categories (i.e., multiple injuries, unspecified injury, multiple body regions, or unspecified).

Table 1. Baseline characteristics of the 8627 patients who died in hospital among 598,137 incident hospital admissions for injury in Sweden, 1998-2004

\begin{tabular}{|c|c|c|}
\hline Characteristic & $\begin{array}{c}\text { Autopsy }(\mathrm{n}=2084) \\
\mathrm{N}(\%)\end{array}$ & $\begin{array}{c}\text { No Autopsy }(\mathrm{n}=6543) \\
\text { N }(\%)\end{array}$ \\
\hline \multicolumn{3}{|l|}{ Age (yrs) } \\
\hline 0-19 & $101(82.1)$ & $22(17.9)$ \\
\hline $20-64$ & $780(72.0)$ & $304(28.0)$ \\
\hline $65-79$ & $531(27.7)$ & $1383(72.3)$ \\
\hline $80+$ & $672(12.2)$ & $4834(87.8)$ \\
\hline \multicolumn{3}{|l|}{ Sex } \\
\hline Men & $1253(28.6)$ & 3131 (71.4) \\
\hline Women & $751(17.3)$ & $3601(82.7)$ \\
\hline \multicolumn{3}{|l|}{ Length of stay (days) } \\
\hline $0-2$ & 1178 (43.5) & $1532(56.5)$ \\
\hline $3-7$ & $444(17.1)$ & $2154(82.9)$ \\
\hline $8+$ & $462(13.9)$ & $2857(86.1)$ \\
\hline \multicolumn{3}{|l|}{ Cause of injury } \\
\hline Cut/pierce & $47(81.0)$ & $11(19.0)$ \\
\hline Drowning & $34(70.8)$ & $14(29.2)$ \\
\hline Fall & $953(14.1)$ & $5814(85.9)$ \\
\hline Fire/flame & $66(64.1)$ & $37(35.9)$ \\
\hline Hot object/scald & $4(28.6)$ & $10(71.4)$ \\
\hline Firearm & $55(91.7)$ & $5(8.3)$ \\
\hline Machinery & $5(62.5)$ & $3(37.5)$ \\
\hline Traffic & $565(78.0)$ & $159(22.0)$ \\
\hline Natural/environment & $16(35.6)$ & $29(64.4)$ \\
\hline Overexertion & $1(5.9)$ & $16(94.1)$ \\
\hline Poisoning & $148(59.7)$ & $100(40.3)$ \\
\hline Struck by/against & $32(68.1)$ & $15(31.9)$ \\
\hline Suffocation & $38(62.3)$ & $23(37.7)$ \\
\hline Other specified & $43(61.4)$ & $27(38.6)$ \\
\hline Other specified, NEC & $5(62.5)$ & $3(37.5)$ \\
\hline Not specified & $41(26.1)$ & $116(73.9)$ \\
\hline Missing & $31(16.1)$ & $161(83.9)$ \\
\hline \multicolumn{3}{|l|}{ Intent of injury } \\
\hline Suicide & $186(67.6)$ & 89 (32.4) \\
\hline Homicide & $61(95.3)$ & $3(4.7)$ \\
\hline Unintentional & $1741(21.8)$ & $6263(78.2)$ \\
\hline Undetermined & $66(70.2)$ & $28(29.8)$ \\
\hline Missing & $30(15.8)$ & $160(84.2)$ \\
\hline
\end{tabular}

NEC, not elsewhere classifiable.
Diagnostic information from autopsies with an autopsy rate of $24.2 \%$ therefore added new specific-injury categories in $7.4 \%(95 \%$ CI $6.8-8.0)$ of all injuryrelated hospital deaths.

In the multivariable analysis of predictive factors for finding a new specific injury at autopsy, cases of burn and firearm injuries were associated with a probability decrease of $92 \%$ (95\% CI 76-97) and 81\% (95\% CI 54-92), respectively, whereas traffic and natural/environment as causes increased the probability of having a new diagnosis category identified at autopsy by $103 \%$ (95\% CI 49-176) and 279\% (95\% CI 23-1071), respectively (Table 2). The multivariable prediction model for finding a new specific diagnosis at autopsy had a c-statistic of 0.881 .

The county (administrative healthcare region) of hospitalization was predictive both for whether autopsy would be performed and for whether a new injury would be found at autopsy, and was therefore included in the multivariable models. A variable indicating the annual number of cases of severe trauma at the hospital to which the patient was primarily admitted did not add predictive capacity to the model beyond that of hospital county, as indicated by the c-statistic.

A longer hospital stay seemed to be associated with a lower probability of autopsy and a reduced probability of finding a new specific-injury category at autopsy. The association remained after multivariable adjustment, but the odds ratio estimate was unstable, with a wide CI. Correlation between length of hospital stay and other variables, such as injury category and injury severity (ICISS), might generate problems in regression analyses, but a variance inflation factor of 1.04 for length of stay did not indicate harmful collinearity.

In $10 \%$ (11 of 116$)$ of the specificinjury categories, addition of autopsy diagnoses reduced the estimated survival risks by at least $0.5 \%$ (Table 3 ). The autopsy rate and mortality were very high among the patients who contributed to these injury categories.

ICISS together with age and sex was highly predictive of hospital mortality (Fig. 1). Addition of autopsy diagnoses resulted in an improved predictive capacity, with an increase in the area under the receiver operating characteristic curve of 0.022 (95\% CI $0.019-0.024)$. When surviving patients with a hospital stay $\leq 1$ day were excluded from the analysis, this 


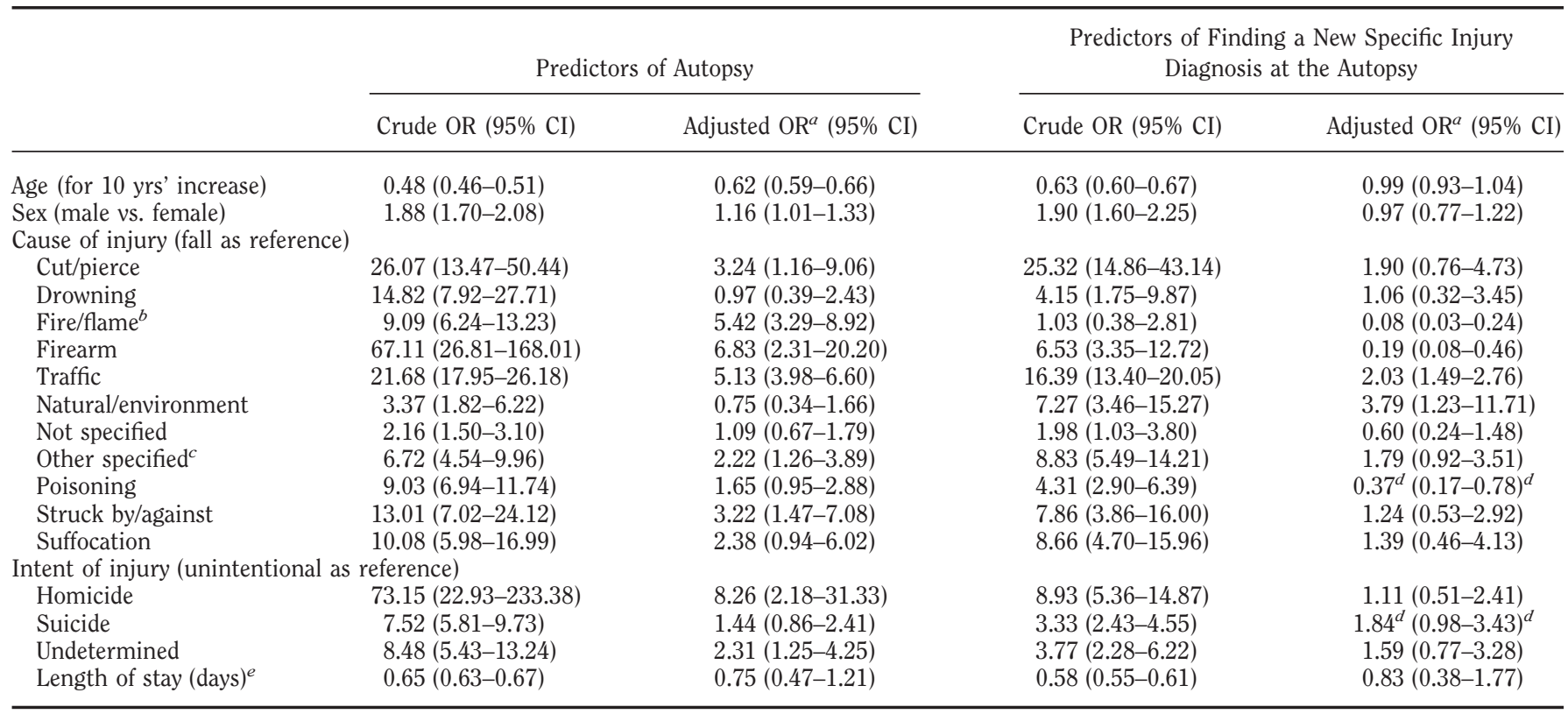

OR, odds ratio; CI, confidence interval.

Prediction models derived from 2084 autopsies in 8627 in-hospital deaths. These deaths occurred among 598,137 incident hospital admissions for injury in Sweden, 1998-2004.

${ }^{a}$ Adjustment for all variables listed in the table are also for injury category (according to the ICD-10 injury mortality diagnosis matrix), International Classification of Diseases Injury Severity Score, and geographical region; ${ }^{b}$ includes category "Hot object/scald."; cincludes categories "Overexertion," "Machinery," and "Other specified, not elsewhere classifiable."; "the estimates for poisoning and suicide may be expected to be attenuated by some degree of collinearity (variance inflation factor 2.76 and 2.57 , respectively); ${ }^{e} \operatorname{logarithm}$ with base 2 , odds ratio, therefore, indicates the effect when the duration of stay is twice as long.

Table 3. Estimated survival risk for different injury categories in 598,137 incident hospital admissions for injury in Sweden, 1998-2004

\begin{tabular}{|c|c|c|c|c|c|c|}
\hline Nature of Injury & Anatomical Region & $\begin{array}{l}\text { Number of } \\
\text { Subjects }^{a}\end{array}$ & $\begin{array}{c}\text { Autopsy } \\
\text { Rate \% (N) }\end{array}$ & $\begin{array}{l}\text { Survival Risk (Excluding } \\
\text { Autopsy Diagnoses) }\end{array}$ & $\begin{array}{l}\text { Survival Risk (Including } \\
\text { Autopsy Diagnoses) }\end{array}$ & $\begin{array}{c}\text { Risk Difference } \\
(95 \% \text { Confidence } \\
\left.\text { Interval }^{b}\right)\end{array}$ \\
\hline Blood vessel & Thorax & 158 & $93.5(58)$ & 0.65 & 0.61 & $0.045(0.020-0.074)$ \\
\hline Crush & $\begin{array}{l}\text { Traumatic brain } \\
\text { injury }\end{array}$ & 175 & $92.3(12)$ & 0.97 & 0.93 & $0.045(0.017-0.078)$ \\
\hline Blood vessel & Abdomen & 93 & $90.9(30)$ & 0.68 & 0.65 & $0.036(0.007-0.071)$ \\
\hline Internal organ injury & Spinal cord & 617 & $60(42)$ & 0.91 & 0.89 & $0.025(0.013-0.038)$ \\
\hline Blood vessel & $\begin{array}{l}\text { Abdomen, lower } \\
\text { back and pelvis }\end{array}$ & 116 & $90.5(19)$ & 0.84 & 0.82 & $0.021(0.000-0.046)$ \\
\hline Fracture & $\begin{array}{l}\text { Traumatic brain } \\
\text { injury }\end{array}$ & 9606 & $74.9(480)$ & 0.95 & 0.93 & $0.021(0.019-0.024)$ \\
\hline Open wound & Thorax & 843 & $95.5(21)$ & 0.98 & 0.97 & $0.009(0.004-0.016$ \\
\hline
\end{tabular}

Survival risk estimates for injury categories based on diagnoses made with and without the aid of autopsy. Results are presented for injury categories where the risk difference between these two diagnostic procedures is at least $0.5 \%$ and the estimate is based on at least ten autopsies.

${ }^{a}$ The number of subjects includes both those classified from hospital discharge diagnoses alone and those classified after addition of autopsy diagnoses; ${ }^{b}$ confidence limits were calculated using a bootstrap procedure and taking into account the dependence between the samples.

difference increased to $0.035(95 \% \mathrm{CI}$ $0.032-0.039)$.

The mean probability of finding a new specific injury given an increased autopsy rate is plotted in Figure 2. This indicated that $25.1 \%(95 \%$ CI $23.0-27.1)$ of the patients who died in hospital would have had at least one new specific-injury category added if all had undergone autopsy. In other words, addition of autopsy diag- noses to hospital discharge diagnoses with the present autopsy rate of $24 \%$ was estimated to reduce misclassification of deaths by $>25 \%$ (i.e., $7.4 \%$ of all deaths were reclassified, as compared with the 


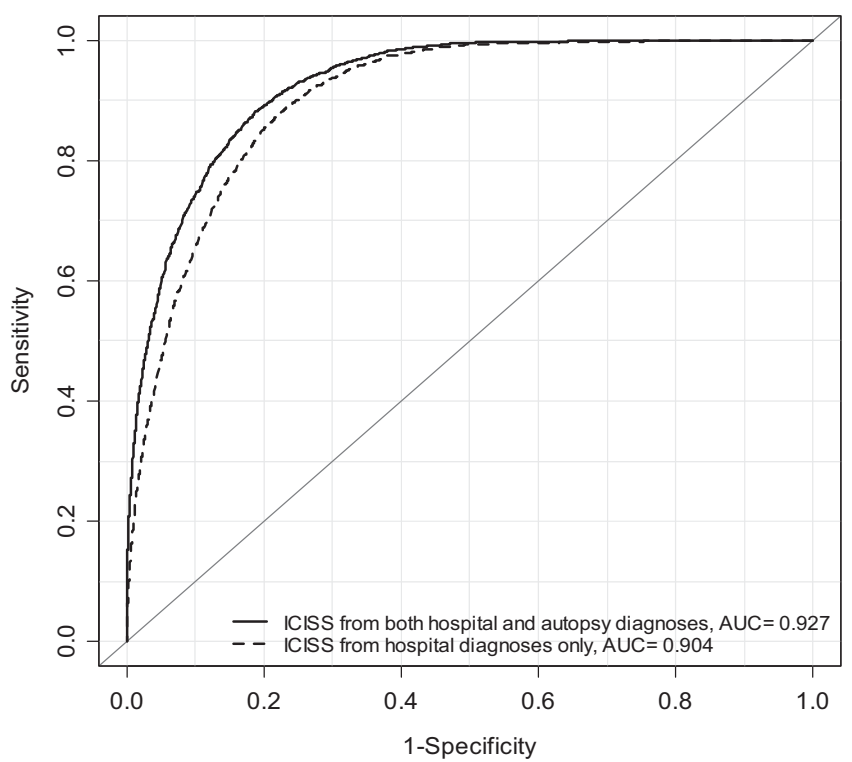

Figure 1. Receiver operating characteristics curves for prediction of hospital mortality from age, sex, and International Classification of Diseases Injury Severity Score (ICISS) (age and ICISS as restricted cubic splines with three nodes) among 168,168 incident injury admissions during the years 20032004. Survival risk ratios (SRRs) for calculation of ICISS were derived in a training dataset $(\mathrm{n}=$ 429,969 from the years 1998-2002). SRRs and ICISS were calculated from hospital discharge diagnoses only and compared with SRRs and ICISS obtained from both hospital and autopsy diagnoses. $A U C$, area under the curve (c-statistic).

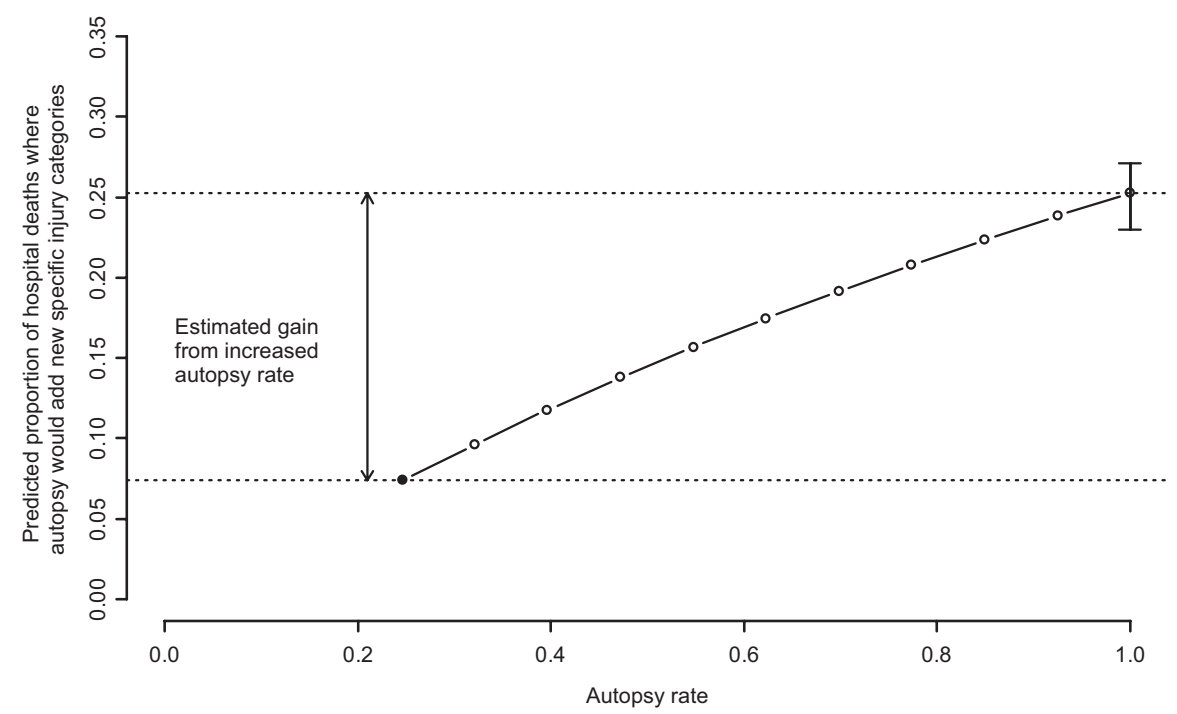

Figure 2. Estimated gain in identification of clinically undiagnosed injuries resulting from an increased autopsy rate. The curve was constructed from prediction models based on patient characteristics (age, sex, injury categories, length of hospital stay, cause of injury, intent of injury, and geographical region) in 2084 autopsies among 8627 in-hospital deaths in Sweden, 1998-2004. Error bars indicate $95 \%$ confidence interval.

estimated total of $25 \%$ of deaths with clinically undiagnosed injuries).

The clinical relevance of these missed injuries is illustrated by their impact on predicted survival (ICISS) among the deaths in which new diagnoses were found at autopsy (Fig. 3). The clinically undetected injuries caused a difference in median predicted survival of $16.9 \%$. This that no survivors with these diagnoses are undetected. Hence, by changing this assumption the proportion of mortality attributable to missed injuries will be reduced.

\section{DISCUSSION}

This study of all hospitalized injuries in Sweden during a 7-year period elucidates the extent of bias of survival risk estimates caused by injuries that are first discovered at autopsy and not registered in hospital discharge records. This effect is evident despite an autopsy rate of only $24 \%$.

There are three major reasons for the discrepancy between hospital and autopsy diagnoses. First, it could represent clinically undiagnosed injuries, which would be a direct indicator of deficiencies in the clinical management in the trauma care system. Second, it could be a result of inaccurate coding of injuries at hospital discharge. This would not be directly related to the clinical management of the patient. Third, it could be due to differences in coding practices between the clinician and the pathologist/forensic examiner. This last possible source of error was dealt with by analyzing injury categories rather than individual injury codes, and by looking only at specificinjury categories. Regardless of the cause of the discrepancy it was found possible to increase the accuracy of risk estimates for hospital survival and consequently accuracy of injury severity measures by merging autopsy data with hospital discharge data. An increase in the autopsy rate would further reduce this bias. Merging hospital and autopsy data can also provide an estimate of the extent of potentially preventable deaths attributable to diagnostic errors (12).

This study illustrates new aspects of the well-known problem of clinically missed injury diagnoses discovered at autopsy $(6,13,14)$. Our focus on the effect of overlooked injuries on survival risk estimates in a population-based perspective stems from the good performance of hospital discharge data compared with trauma registers (15) and the excellent opportunity for injury severity adjustments that is offered by injury-specific survival risk estimates $(3,4)$. Even a small number of misclassified hospital deaths can, however, substantially reduce the estimated survival.

Missed injuries as a major clinical problem were described in pivotal articles 


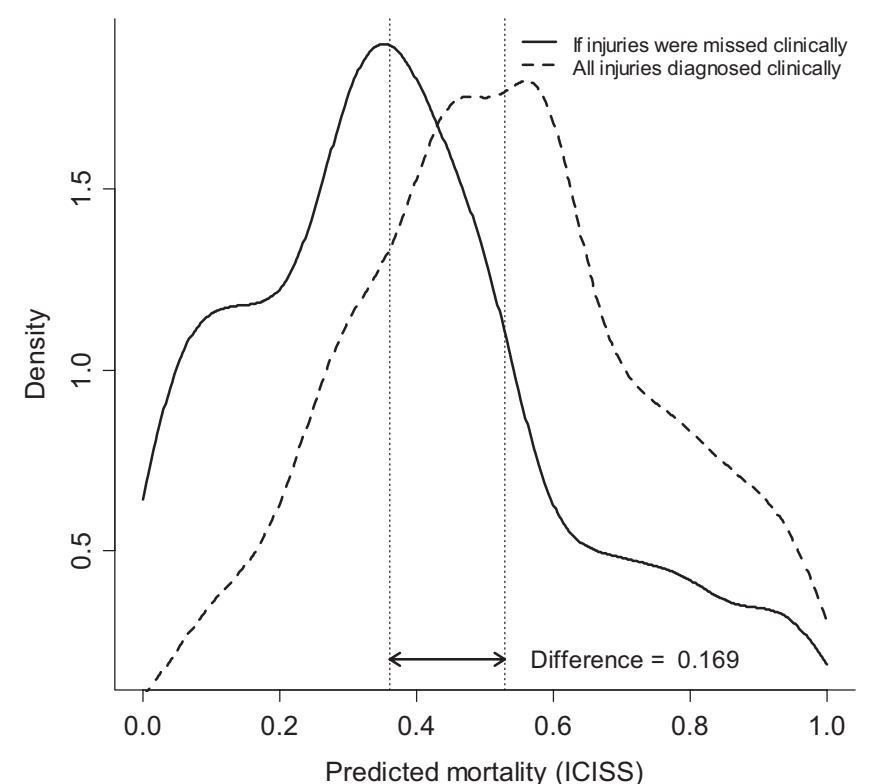

Figure 3. Kernel density plot with Gaussian smoothing that illustrates the distribution of predicted survival (International Classification of Diseases Injury Severity Score [ICISS]) among 638 deaths where new injuries were found at autopsy. ICISS was calculated from survival risk ratios based solely on hospital discharge diagnoses and compared with ICISS from survival risk ratios also including autopsy diagnoses. The difference in medians is used to estimate the maximum proportion of injury deaths attributable to clinically undiagnosed injuries.

that laid the foundation for the organization of modern trauma care $>25$ years ago $(16,17)$. Although a well-known phenomenon in clinical medicine, the pattern and extent of missed injuries vary depending on the setting and the time period under study $(7,18-24)$. It is likely that the proportion of missed injuries has decreased over time, as a result of implementation of structured clinical assessment of trauma victims (25) and improved diagnostic procedures (26). Nevertheless, in a study on traumarelated deaths in an intensive care unit by Ong et al (14), new major diagnoses were found in 19\% of the autopsies. This corresponds to our estimate that new specific injuries would be disclosed in $25 \%$ given a $100 \%$ autopsy rate among all hospitalizations or deaths because of trauma in Sweden.

An obvious question to consider is the possible impact of missed injuries on the clinical outcome. This is a difficult issue, as it involves determining the causal relation between different injuries and mortality. The commonly used strategy in clinical audit is retrospective chart review by expert panels (27). The alternative approach that we used is based on a comparison between predicted survival based on SRRs including autopsy diagnoses, and that based on SRRs from hospital discharge diagnoses alone. From this comparison, the proportion of potentially preventable deaths can be estimated. This method is appealing as it also takes into account the severity of other injuries and not merely the severity of the clinically undiagnosed injury. But it also involves several assumptions that need to be considered. The most critical assumption is that no patients survive with clinically undiagnosed injuries of the same type as those discovered at autopsy. This assumption is not unreasonable. SRRs generated under this supposition improve mortality prediction. The injuries that are only detected at autopsy are associated with a high mortality and they also seem severe enough from a clinical viewpoint to support such an assumption. Nevertheless, any departure from this assumption will lower the estimated proportion of potentially preventable deaths associated with clinically undiagnosed injuries. Failure to code for these injuries at hospital discharge, even when they have been recognized and treated clinically, could also bias the estimated proportion of potentially preventable deaths. If accurate hospital discharge coding can be maintained, the estimate of mortality attributable to missed injuries will be a potential indicator of clinically important diagnostic failures. The average number of deaths attributable to diagnostic failures per hospital is small enough for them to be able to pass unrecognized as a recurring and significant problem in routine clinical audit. A possible way to identify specific and systematic deficiencies in diagnostic procedures and clinical management would be to perform a structured audit of the cases identified in this study.

The results from this study serve as a reminder of some of the possible consequences of a decreasing autopsy rate (28). Survival estimates for the majority of injury categories were unaffected by the addition of autopsy data. This might be due to the comparatively low autopsy rate. According to the prediction model, $\sim 25 \%$ of the subjects would have had new specific injuries identified if all deaths had been subjected to autopsy. However, it is unclear whether survival estimates for other injury categories than those identified in our study would be affected if the autopsy rate increased.

The most pronounced effect on injuryspecific survival was seen for intraabdominal injuries, where addition of autopsy results reduced the estimated chance of survival from $83 \%$ to $69 \%$. This finding confirms the fact that the diagnosis of intra-abdominal injuries remains a difficult issue in trauma management despite increased use of computerized tomography and other imaging techniques (29-31).

Specific strengths of this study include the population-based approach, with inclusion of essentially all hospitalized injuries in Sweden during a 7-year period. We are not aware of any other study with a similar design. Merging autopsy data with clinical data may be prohibited in other settings (6). The ability to link autopsy data to clinical data by unique personal identification numbers offers a powerful way to estimate and reduce misclassification of injury deaths. Multivariable modeling also takes into account notable regional differences in the propensity to perform autopsy, which should therefore increase the external validity of the results.

The study has some potential limitations in addition to those already discussed. The prediction model for diagnosing new specific injuries at autopsy can only be derived from nonautopsied patients. Its validity can be questioned when applied to patients not subjected to autopsy. There is unfortunately no way of validating this model reliably, but the model has some notable properties that lend support to its credibility. Exactly the 
same predictors were found to be predictive for identifying patients subjected to autopsy. At the same time, the distribution of probabilities of finding a new specific injury at autopsy among the autopsied patients (where the model was developed) was similar to that found among patients not subjected to autopsy (where the model was applied). In a substantial part of the population of autopsied patients the probability of finding a new specific injury at autopsy is low. The model would have been less credible if low probabilities of finding a new injury had not been well represented in this population.

In conclusion, survival estimates for some types of abdominal, thoracic, and central nervous system injuries were notably reduced by adding autopsy diagnoses, and this addition also generated more accurate mortality prediction models using ICISS. At most, $6.5 \%$ of all injury deaths were attributable to clinically undiagnosed injuries. Merging hospital discharge data and autopsy data is an effective way of improving the accuracy of injury categorization and mortality prediction.

\section{ACKNOWLEDGMENTS}

We acknowledge the valuable opinions provided by Margaret Warner, National Center for Health Statistics, Centers for Disease Control and Prevention, USA, on the manuscript; and the expert opinions by Johan Lindbäck on $\mathrm{R}$ programming and Sören Gustafsson on SAS programming, both from the Uppsala Clinical Research Center. We also thank Mrs. Maud Marsden for language revision.

\section{REFERENCES}

1. Branas CC, MacKenzie EJ, Williams JC, et al: Access to trauma centers in the United States. JAMA 2005; 293:2626-2633

2. Utter GH, Victorino GP, Wisner DH: Interhospital transfer occurs more slowly for el- derly acute trauma patients. $J$ Emerg Med 2008; 35:415-420

3. Clark DE, Winchell RJ: Risk adjustment for injured patients using administrative data. J Trauma 2004; 57:130-140; discussion 140

4. Stephenson S, Henley G, Harrison JE, et al: Diagnosis based injury severity scaling: Investigation of a method using Australian and New Zealand hospitalisations. Inj Prev 2004; 10:379-383

5. Fingerhut LA, Warner M: The ICD-10 injury mortality diagnosis matrix. Inj Prev 2006; $12: 24-29$

6. Marx WH, Simon HM, Jumbelic M, et al: Severity of injury is underestimated in the absence of autopsy verification. $J$ Trauma 2004; 57:46-49; discussion 49-50

7. Sharma BR, Gupta M, Bangar S, et al: Forensic considerations of missed diagnoses in trauma deaths. J Forensic Leg Med 2007; 14:195-202

8. Sharma BR, Gupta M, Harish D, et al: Missed diagnoses in trauma patients vis-a-vis significance of autopsy. Injury 2005; 36:976-983

9. National Center for Health Statistics: International Collaborative Effort (ICE) on Injury Statistics: Overview and implications. In: Atlanta: Centers for Disease Control and Prevention, 2007

10. Recommended framework for presenting injury mortality data. MMWR Recomm Rep 1997; 46:1-30

11. R Development Core Team: R: A Language and Environment for Statistical Computing. In. Version 2.5.1 ed: R Foundation for Statistical Computing, 2007

12. Martin BT, Fallon WF Jr, Palmieri PA, et al: Autopsy data in the peer review process improves outcomes analysis. J Trauma 2007; 62:69-73; discussion 73

13. Enderson BL, Maull KI: Missed injuries. The trauma surgeon's nemesis. Surg Clin North Am 1991; 71:399-418

14. Ong AW, Cohn SM, Cohn KA, et al: Unexpected findings in trauma patients dying in the intensive care unit: Results of 153 consecutive autopsies. J Am Coll Surg 2002; 194: 401-406

15. McCarthy ML, Shore AD, Serpi T, et al: Comparison of Maryland hospital discharge and trauma registry data. $J$ Trauma 2005; 58: 154-161

16. West JG, Trunkey DD, Lim RC: Systems of trauma care. A study of two counties. Arch Surg 1979; 114:455-460
17. Baker CC, Oppenheimer L, Stephens B, et al: Epidemiology of trauma deaths. Am J Surg 1980; 140:144-150

18. Chan RN, Ainscow D, Sikorski JM: Diagnostic failures in the multiple injured. J Trauma 1980; 20:684-687

19. Hamdan TA: Missed injuries in casualties from the Iraqi-Iranian war: A study of 35 cases. Injury 1987; 18:15-17

20. Albrektsen SB, Thomsen JL: Detection of injuries in traumatic deaths. The significance of medico-legal autopsy. Forensic Sci Int 1989; 42:135-143

21. Enderson BL, Reath DB, Meadors J, et al: The tertiary trauma survey: A prospective study of missed injury. J Trauma 1990; 30: 666-669; discussion 669-670

22. Frawley PA: Missed injuries in the multiply traumatized. Aust N Z J Surg 1993; 63: 935-939

23. Hirshberg A, Wall MJ Jr, Allen MK, et al: Causes and patterns of missed injuries in trauma. Am J Surg 1994; 168:299-303

24. Houshian S, Larsen MS, Holm C: Missed injuries in a level I trauma center. J Trauma 2002; 52:715-719

25. van Olden GD, Meeuwis JD, Bolhuis HW, et al: Clinical impact of advanced trauma life support. Am J Emerg Med 2004; 22:522-525

26. Poletti PA, Wintermark M, Schnyder P, et al: Traumatic injuries: Role of imaging in the management of the polytrauma victim (conservative expectation). Eur Radiol 2002; 12: 969-978

27. Chiara O, Cimbanassi S, Pitidis A, et al: Preventable trauma deaths: From panel review to population based-studies. World J Emerg Surg 2006; 1:12

28. Burton JL, Underwood J: Clinical, educational, and epidemiological value of autopsy. Lancet 2007; 369:1471-1480

29. Sung CK, Kim KH: Missed injuries in abdominal trauma. J Trauma 1996; 41: 276-282

30. Sirlin CB, Brown MA, Deutsch R, et al: Screening US for blunt abdominal trauma: Objective predictors of false-negative findings and missed injuries. Radiology 2003; 229:766-774

31. Yamazaki K, Shiotani S, Ohashi N, et al: Comparison between computed tomography (CT) and autopsy findings in cases of abdominal injury and disease. Forensic Sci Int 2006; 162:163-166 